\title{
BMJ Open Excess burden of age-associated comorbidities among people living with HIV in British Columbia, Canada: a population-based cohort study
}

Ni Gusti Ayu Nanditha (D , ${ }^{1,2}$ Adrianna Paiero, ${ }^{1}$ Hiwot M Tafessu, ${ }^{1}$ Martin St-Jean, ${ }^{1}$ Taylor McLinden, ${ }^{1}$ Amy C Justice, ${ }^{3,4}$ Jacek Kopec, ${ }^{5,6}$ Julio S G Montaner,, ${ }^{1,2}$ Robert S Hogg, ${ }^{1,7}$ Viviane D Lima ${ }^{1,2}$

To cite: Nanditha NGA, Paiero A, Tafessu HM, et al. Excess burden of age-associated comorbidities among people living with HIV in British Columbia, Canada: a populationbased cohort study. BMJ Open 2021;11:e041734. doi:10.1136/ bmjopen-2020-041734

- Prepublication history for this paper is available online. To view these files, please visit the journal online (http://dx.doi org/10.1136/bmjopen-2020041734).

Preliminary results were presented by NGAN at the 2019 Conference on Retroviruses and Opportunistic Infections (CROI) Seattle, USA, from $4^{\text {th }}$ to $7^{\text {th }}$ March 2019 (abstract 2540), receiving the New Investigator Award.

Received 16 June 2020 Revised 10 December 2020 Accepted 18 December 2020

Check for updates

(C) Author(s) (or their employer(s)) 2021. Re-use permitted under CC BY-NC. No commercial re-use. See rights and permissions. Published by BMJ.

For numbered affiliations see end of article.

Correspondence to Dr Viviane D Lima; vlima@bccfe.ca

\section{ABSTRACT}

Objectives As people living with HIV (PLWH) live longer, morbidity and mortality from non-AIDS comorbidities have emerged as major concerns. Our objective was to compare prevalence trends and age at diagnosis of nine chronic age-associated comorbidities between individuals living with and without HIV.

Design and setting This population-based cohort study used longitudinal cohort data from all diagnosed antiretroviral-treated PLWH and 1:4 age-sex-matched HIVnegative individuals in British Columbia, Canada.

Participants The study included 8031 antiretroviraltreated PLWH and 32124 HIV-negative controls (median age 40 years, $82 \%$ men). Eligible participants were $\geq 19$ years old and followed for $\geq 1$ year during 2000 to 2012 . Primary and secondary outcome measures The presence of non-AIDS-defining cancers, diabetes, osteoarthritis, hypertension, Alzheimer's and/or non-HIVrelated dementia, cardiovascular, kidney, liver and lung diseases were identified from provincial administrative databases. Beta regression assessed annual age-sexstandardised prevalence trends and Kruskal-Wallis tests compared the age at diagnosis of comorbidities stratified by rate of healthcare encounters.

Results Across study period, the prevalence of all chronic age-associated comorbidities, except hypertension, were higher among PLWH compared with their communitybased HIV-negative counterparts; as much as 10 times higher for liver diseases ( $25.3 \%$ vs $2.1 \%$, $p$ value $<0.0001$ ). On stratification by healthcare encounter rates, PLWH experienced most chronic age-associated significantly earlier than HIV-negative controls, as early as 21 years earlier for Alzheimer's and/or dementia.

Conclusions PLWH experienced higher prevalence and earlier age at diagnosis of non-AIDS comorbidities than their HIV-negative controls. These results stress the need for optimised screening for comorbidities at earlier ages among PLWH, and a comprehensive HIV care model that integrates prevention and treatment of chronic ageassociated conditions. Additionally, the robust methodology developed in this study, which addresses concerns on the use of administrative health data to measure prevalence and incidence, is reproducible to other settings.
Strengths and limitations of this study

- This population-based cohort study described and compared trends in prevalence and age at diagnosis of nine chronic age-related comorbidities among people living with HIV (PLWH) and a population of age-sex-matched HIV-negative individuals from the same geographical setting where universal health coverage is available.

- The strengths of this study include its populationbased nature, a large number of participants, a 12-year observation span and confidential linkages among several provincial healthcare databases, which enabled an extensive review of individuals' healthcare utilisation, including physician visits, hospitalisations and prescription medications.

- Our study employed robust methodological approaches to address potential biasses when comparing PLWH to HIV-negative individuals including a judicious lookback observation window, considering differences in the demographics and healthcare engagement level of these populations and the employment of sound case-finding algorithms of comorbidities.

- This study used administrative health data, which, although an important source of information for empirical decision-making and medical research, were not collected for research purposes; these data may be prone to coding errors and did not contain key socioeconomic and lifestyle information.

- Ultimately, our study illustrates a vigorous and replicable methodology for studies of disease burden, particularly those using administrative health data, and it provides evidence for a more integrated HIV care model that reflects the present intersectionality of HIV and chronic diseases, and best suits the evolving healthcare needs of ageing PLWH.

\section{INTRODUCTION}

Advances in antiretroviral therapy (ART) have transformed HIV from a rapidly fatal infection to a manageable chronic condition. ${ }^{1}$ As a result, in settings where ART is 
highly accessible, the life expectancy of people living with HIV (PLWH) has dramatically improved and now approaches that of the general population. ${ }^{23}$ This progress has corresponded to a demographic shift among PLWH in developed nations. In British Columbia (BC), Canada, for instance, the proportion of diagnosed PLWH older than 50 years of age has increased from $47.0 \%$ in 2013 to $60.4 \%$ in $2018 .^{45}$

As PLWH age, they face novel challenges including higher incidence of age-associated conditions, ${ }^{6}$ and experience excess morbidity and mortality from these conditions. ${ }^{7-10}$ It has been reported that compared with their HIV-negative counterparts, PLWH have a greater risk of developing chronic age-associated comorbidities, including non-AIDS-related cancers, cardiovascular, lung, bone, kidney and liver diseases; ${ }^{11}$ as well as geriatric syndromes, such as frailty and multimorbidity. ${ }^{12}$ Whether the impact of HIV on PLWH's risk of age-associated comorbidities progressively increases (ie, 'accelerated ageing') or holds firm (ie, 'accentuated ageing') as age increases, ${ }^{13} 14$ remains a matter of great interest. However, as critics contended, studies exploring HIV and ageing frequently failed to employ appropriate control groups, ${ }^{15}$ or consider key confounders including differences in the demographics between PLWH and the control groups. ${ }^{13}$ This reality underlines the need for more carefully examined epidemiological evidence to identify existing gaps in care for ageing PLWH and an approach that best addresses their present and future healthcare needs.

Administrative data, collected to facilitate payment associated with healthcare encounters, has become an indispensable tool in contemporary health research. In measuring disease frequency using administrative data, the amount of lookback time to search for relevant healthcare encounters is crucial in ensuring adequate data observability to produce reliable prevalence and incidence estimates. ${ }^{16}$ With longitudinal data, fixed-size, as opposed to all-time, lookback observation window has been found to provide more stable annual estimates over time by allowing similar amounts of historical data available for each year. ${ }^{16}$ Previous administrative data-based studies exploring HIV and ageing have failed to account for this observability when estimating annual prevalence and incidence of chronic diseases among PLWH. ${ }^{17-21}$ In addition, some studies also used more lenient casefinding algorithms (eg, identifying comorbidity diagnoses from a single physician visit), thus not addressing the nature of administrative data, and its susceptibility to coding errors. ${ }^{1821}$

In this study, we used a population-based cohort with key linkages to provincial health databases in BC to describe and compare trends in prevalence and age at first diagnosis of selected chronic age-associated comorbidities among PLWH and a population of age-and-sex-matched HIVnegative controls. We employed a robust methodological approach incorporating a fixed-size lookback observation window, and vigorous case-finding algorithms to improve the ascertainment of comorbidity diagnoses. To further elucidate these outcomes, unlike previous studies, we considered key confounders by stratifying the prevalence of comorbidities by PLWH's ethnicity, history of injection drug use (IDU) and sex at birth, and we considered differences in healthcare engagement between PLWH and HIV-negative individuals when comparing the age at diagnosis of comorbidities.

\section{METHODS}

\section{Study setting}

In $\mathrm{BC}, \mathrm{ART}$ and routine laboratory monitoring (eg, viral load and CD4 cell counts) are provided free of charge (no copayments or deductibles) to all HIV-diagnosed residents. The BC Centre for Excellence in HIV/AIDS Drug Treatment Program (BC-CfE DTP) has been responsible for the distribution of ART in the province since 1992. ART eligibility is in accordance with BC's HIV therapeutic guidelines. $^{22}$

\section{Data sources}

Data were obtained from the Comparative Outcomes And Service Utilization Trends (COAST) study, comprising longitudinal de-identified individual-level data on all diagnosed adult PLWH in the province and a $10 \%$ random representative sample of BC's general population followed from 1996 to 2013. The COAST study confidentially links two provincial data sources in BC: (1) the BC-CfE DTP, which provides demographic, treatmentrelated and clinical information of all known PLWH $;{ }^{23}$ and (2) Population Data BC, ${ }^{24-29}$ which houses data from various provincial administrative health databases for all $\mathrm{BC}$ residents. The COAST cohort and linkages between the aforementioned data sources have been described elsewhere. $^{30}$

\section{Study design}

In this population-based matched-cohort study, eligible participants were $\geq 19$ years old and followed for at least 1 year between 1 January 2001 and 31 December 2012. ART-treated PLWH were randomly matched at a 1:4 ratio to HIV-negative individuals from the COAST cohort by birthyear and sex at birth. For PLWH, baseline was the latest among the following dates: known positive HIV serostatus, $19^{\text {th }}$ birthday, 5 years since the first administrative date (earliest date of either provincial Medical Service Plan (MSP) registration or the first identified healthcare encounter), or 1 January 2001. For HIVnegative controls, the same baseline date as their matched PLWH was assigned. The end of the observation period was the earliest of either the date of death, date of loss-tofollow-up, or 31 December 2012.

\section{Selected comorbidities}

Nine chronic age-associated comorbidities, prevalent in the wider BCpopulation and among $\mathrm{PLWH},{ }^{1131}$ were examined: cardiovascular (CVD), kidney, liver, chronic obstructive pulmonary disease (COPD), non-AIDS-defining 
cancers (hereinafter referred to as cancers), diabetes, osteoarthritis, hypertension and Alzheimer's and/or non-HIV-related dementia (Alzheimer's/dementia). In both study populations, comorbidities were identified by the same published case-finding algorithms using International Classification of Disease Ninth and Tenth Revisions (ICD 9/10) diagnosis codes and Canada-wide Drug Identification Numbers. These algorithms were applied to the following BC's administrative health databases: (1) the Discharge Abstract Database, which captures hospital discharges and deaths of inpatients and day-surgery patients from acute-care hospitals; (2) MSP billings database, which captures medically necessary outpatient services provided by physicians, laboratory and diagnostic procedures; and (3) PharmaNet database, which captures all prescription drugs dispensed by community and outpatient pharmacies. Additionally, the BC Cancer Agency Registry identified cancer cases other than Kaposi sarcoma, non-Hodgkin's lymphoma and cervical cancer. Online supplemental table 1 provides detail on the selected comorbidities and case-finding algorithms.

\section{Outcome variables}

Our outcomes were period prevalence (hereinafter referred to as prevalence) and age at diagnosis of comorbidities. We reported the annual age-sex-standardised prevalence, using Canada's 2006 census as a reference population, ${ }^{32}$ and the 12 -year prevalence. Annual prevalence was the proportion of population present for $\geq 1$ hour in a particular year with an existing condition identified within a 5-year observation window (ie, at any point that year and/or within 4 years prior). Cumulatively, the proportion of the overall study population who ever had a prevalent condition during 2001 to 2012, as defined above, constituted the 12-year prevalence. Among PLWH, we subanalysed the 12-year prevalence of each comorbidity stratified by ethnicity (Caucasian, non-Caucasian, unknown), history of injection drug use (IDU, non-IDU, unknown) and sex at birth (male, female). Note that following age-restrictions in the case-finding algorithms, the denominator in the prevalence calculation for hypertension, COPD and Alzheimer's/dementia was the total number of individuals older than 20,35 and 40 years, respectively.

The age at first diagnosis for each comorbidity was ascertained for each individual considering a run-in period of 5 years, during which no diagnosis of the named comorbidity should be detected. To address differences in healthcare engagement between PLWH and HIV-negative individuals, the distribution of age at diagnosis of a comorbidity was stratified based on the rate of healthcare encounters preceding the diagnosis. This rate was calculated as the number of healthcare encounters prior to an individual's diagnosis of a condition, including all hospitalisations and physician visits, divided by the number of years of follow-up. Multiple healthcare encounters attributed to the same condition at the same service location within 24 hours of each other were regarded as one encounter. The distribution of these rates among PLWH was used to create four ordinal categories: from minimum value to $25^{\text {th }}$ percentile (lowest category); $25^{\text {th }}$ percentile to median (lower); median to $75^{\text {th }}$ percentile (higher); and $75^{\text {th }}$ percentile to maximum value (highest). Group matching was performed, resulting in the inclusion of all PLWH along with HIV-negative controls with comparable rates of healthcare encounters.

\section{Statistical analysis}

Categorical variables were compared using the Fisher's exact test and continuous variables were compared using the Kruskal-Wallis test. The non-linear trends of the agesex-standardised prevalence in each population group across calendar years (2001 to 2012) were examined using generalised non-linear models assuming a beta distribution and a logit link function (ie, beta regression). ${ }^{33} \mathrm{We}$ modelled the prevalence of each comorbidity separately in PLWH and in HIV-negative controls, with year as an explanatory variable. Restricted maximum likelihood was used to estimate parameters in these models. The cumulative rate of healthcare encounters was summarised using Poisson regression. All p values are two-sided and the level of significance was set at $5 \%$. Analyses were performed SAS V.9.4 (SAS Institute, Cary, North Carolina, USA) and R Software V.3.5.2 (R Core Team, Vienna, Austria).

\section{Patient and public involvement}

Patients or the public were not involved in the design, conduct, reporting or dissemination plans of our research.

The study complies with the BC Freedom of Information and Protection of Privacy Act (FIPPA) and did not require informed consent as it is conducted retrospectively for research and statistical purposes only using anonymised data.

\section{RESULTS}

\section{Study population}

Of the 528859 unique individuals in the COAST cohort, 8031 PLWH and 32124 HIV-negative controls constituted our final analytical sample. This stepwise selection process is detailed in online supplemental figure 1. The two groups were identical regarding the median age at baseline (40 years, $\left(25^{\text {th }}\right.$ to $75^{\text {th }}$ percentile: 34 to 47$)$ ) and sex (82\% male), but differed slightly in the median follow-up time (9 years ( 5 to 12 ) for PLWH vs 11 years (6 to 12) for HIV-negative controls; table 1.

\section{Prevalence of comorbidities}

Unadjusted 12-year prevalence

PLWH experienced a greater disease burden compared with HIV-negative controls, driven by significantly higher prevalence of most chronic age-associated comorbidities throughout the study period (figure 1). Exceptions included hypertension (significantly higher in HIVnegative controls; $21.2 \%$ vs $17.9 \%$ ) and osteoarthritis (no significant difference). PLWH experienced 10 times 
Table 1 Descriptive baseline characteristics of a 1:4 matched analytical sample of 8031 PLWH and 32124 HIV-negative individuals in British Columbia, Canada (2001 to 2011)

\begin{tabular}{|c|c|c|c|}
\hline \multirow[b]{2}{*}{ Variables } & & \multirow{2}{*}{$\begin{array}{l}\text { PLWH } \\
n=8031\end{array}$} & \multirow{2}{*}{$\begin{array}{l}\text { HIV-negative } \\
n=32124\end{array}$} \\
\hline & & & \\
\hline \multirow[t]{2}{*}{ Sex at birth, $n(\%)$} & Male & $6612(82)$ & 26448 (82) \\
\hline & Female & $1419(18)$ & $5676(18)$ \\
\hline \multirow[t]{4}{*}{ Age at baseline, $\mathrm{n}(\%)$} & $<30$ & $934(12)$ & $3746(12)$ \\
\hline & $30-39$ & $2861(36)$ & $11397(35)$ \\
\hline & $40-49$ & $2786(35)$ & $11177(35)$ \\
\hline & $\geq 50$ & $1450(18)$ & $5804(18)$ \\
\hline \multicolumn{2}{|c|}{ Age at baseline (years), median (Q1 to Q3) } & 40 (34 to 47$)$ & 40 (34 to 47$)$ \\
\hline \multicolumn{2}{|c|}{ Follow-up time (years), median (Q1 to Q3) ${ }^{\star}$} & $9(5$ to 12$)$ & 11 (6 to 12$)$ \\
\hline \multirow[t]{3}{*}{ History of injection drug use, $\mathrm{n}(\%)$} & IDU & $3075(38)$ & Not available \\
\hline & Non-IDU & $2829(35)$ & \\
\hline & Unknown & $2127(26)$ & \\
\hline \multirow[t]{3}{*}{ Ethnicity, n (\%) } & Caucasian & $3028(38)$ & Not available \\
\hline & Non-Caucasian & $1580(20)$ & \\
\hline & Unknown & $3423(43)$ & \\
\hline
\end{tabular}

Note: PLWH: people living with HIV; Q1 to Q3: $25^{\text {th }}$ to $75^{\text {th }}$ percentiles; IDU: PLWH with a history of injection drug use; Non-IDU: PLWH without a history of injection drug use.

*Significant difference in the follow-up time between PLWH and HIV-negative controls ( $p$ value $<0.05)$.

\section{(A) PLWH}

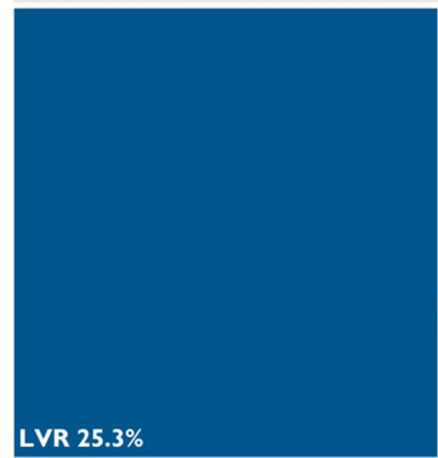

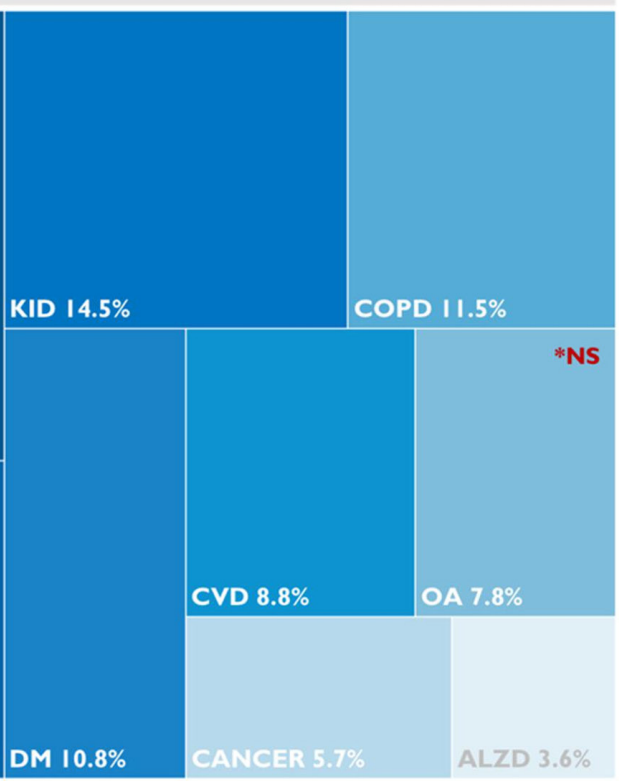

\section{(B) HIV-Negative}

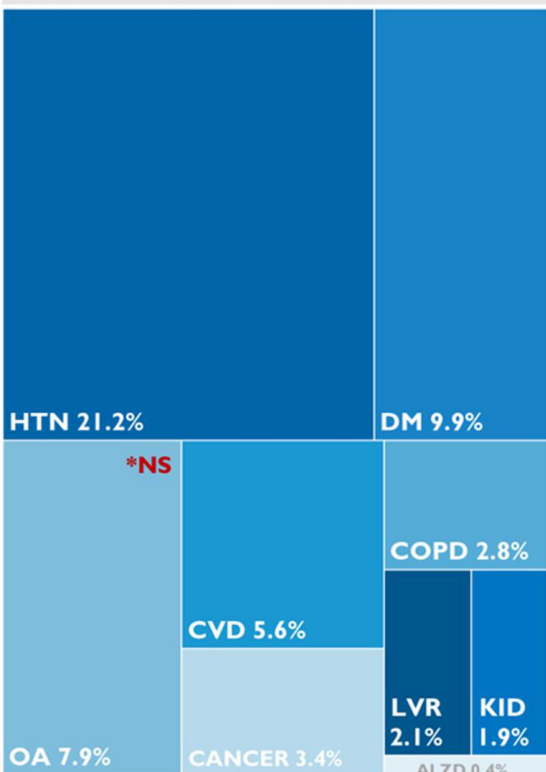

Figure 1 Treemaps of unadjusted 12-year prevalence of selected comorbidities throughout the study period; prevalence in PLWH and HIV-negative controls were significantly different ( $p$ value $<0.0001)$, unless otherwise specified. Note: PLWH, people living with HIV; unless stated, there is a significant difference observed between prevalence in PLWH and HIV-negative controls ( $p$ value $<0.0001$ ); NS, non-significant difference observed between prevalence in PLWH and HIV-negative controls ( $p$ value $>0.05$ ); ALZD, Alzheimer's and/or non-HIV-related dementia (denominator included only individuals aged 40 years and older); CANCER, non-AIDS-defining cancer; COPD, chronic obstructive pulmonary disease (denominator included only individuals aged 35 years and older); CVD, cardiovascular diseases; DM, diabetes mellitus; HTN, hypertension; KID, kidney diseases; LVR, liver diseases; OA, osteoarthritis. 
(A) Ethnicity

30

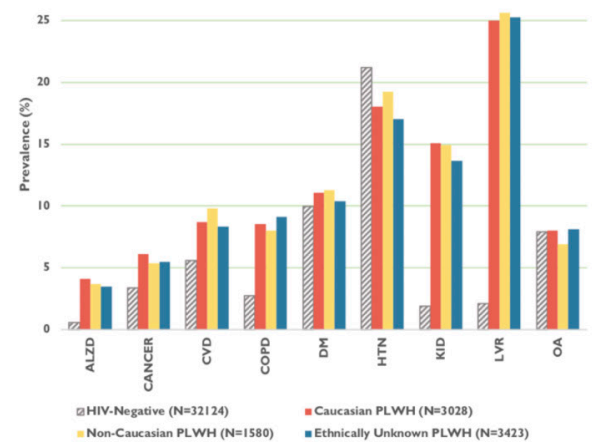

(B) History of injection drug use

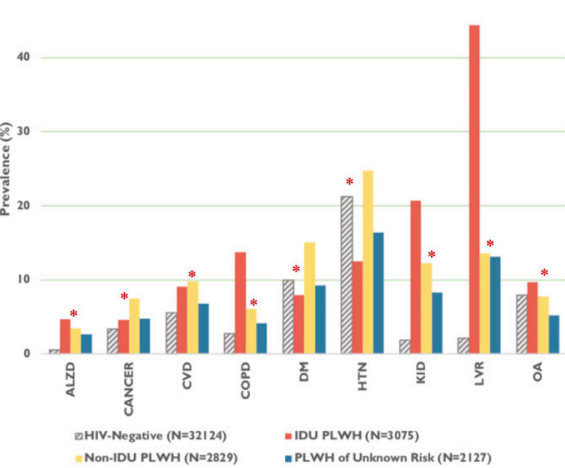

(C) Sex at birth

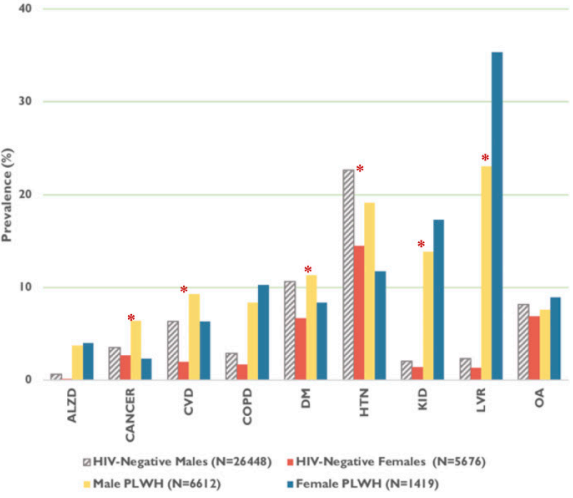

Figure 2 Results of subanalysis of unadjusted 12-year prevalence of selected comorbidities among PLWH, stratified by ethnicity, history of injection drug use and sex at birth. Note: PLWH, people living with HIV; IDU PLWH, PLWH with a history of injection drug use; Non-IDU PLWH, PLWH without a history of injection drug use; ALZD, Alzheimer's and/or non-HIV-related dementia; CANCER, non-AIDS-defining cancer; COPD, chronic obstructive pulmonary disease; CVD, cardiovascular diseases; DM, diabetes mellitus; HTN, hypertension; KID, kidney diseases; LVR, liver diseases; OA, osteoarthritis; ${ }^{*}$, significant difference in the prevalence of comorbidities among PLWH across IDU history and/or sex at birth groups ( $p$ value $<0.05)$. Vertical scales differ for each graph for illustration purposes.

the burden of liver disease compared with HIV-negative controls (25.3\% vs $2.1 \%)$, the greatest disparity among all comorbidities. The most common chronic age-associated comorbidities were liver diseases for PLWH and hypertension for HIV-negative individuals.

Subanalyses among PLWH indicated that the prevalence of all age-associated comorbidities did not vary by ethnicity, but varied greatly by history of IDU and sex at birth (figure 2). Prevalence of COPD, liver and kidney diseases, for instance, were substantially elevated among PLWH with a history of IDU. Liver and kidney diseases were also significantly more prevalent among female compared with male PLWH.

\section{Age-sex-standardised annual prevalence trends}

Consistent with the 12-year prevalence, annually across the study period, the age-sex standardised prevalence of all comorbidities, except hypertension, were higher among PLWH compared with their HIV-negative counterparts (figure 3; data in online supplemental table 2). For cancers, diabetes and osteoarthritis, while prevalence trends were predominantly higher among PLWH, apparent overlaps with the trends in HIV-negative controls, considering the $95 \%$ CIs, should be noted. Our beta regression models (online supplemental table 3) indicated significantly increasing prevalence trends over the study period for most chronic age-associated diseases, with no significant trends observed for Alzheimer's/ dementia and kidney diseases (in PLWH), osteoarthritis (in HIV-negative controls) and CVD (in both groups).

\section{Age at diagnosis of comorbidities}

Unadjusted age at diagnosis

The age at diagnosis of all chronic age-associated comorbidities, except diabetes and hypertension, were significantly lower among PLWH when compared with HIV-negative controls (figure 4; data in online supplemental table 4). Comparing the median age at diagnosis, the largest difference was for Alzheimer's/dementia, where PLWH were diagnosed 12.5 years earlier than their HIV-negative controls, followed by kidney (9.5 years), COPD (6 years) and liver disease ( 5 years $)$.

Age at diagnosis of comorbidities stratified by rate of healthcare encounters

Online supplemental table 5 outlines the distribution of healthcare encounter rates prior to all comorbidity diagnoses, where PLWH had between 2 to 3.2 times the number of healthcare encounters per person-year than HIV-negative controls. When compared exclusively to HIV-negative controls with similar healthcare encounter rates, PLWH were still diagnosed with cancer, COPD, CVD, kidney and liver diseases at significantly younger ages; between 1 to 10 years earlier (figure 4); data in (online supplemental table 6). For Alzheimer's/dementia, which had the largest difference in the unadjusted median age at diagnosis, stratification by healthcare encounter rates uncovered an even larger difference, between 14 to 21 years earlier. However, within the highest healthcare utilisation group, this difference was not observed. The significant difference in age at diagnosis of osteoarthritis also disappeared upon stratification. Conversely, for diabetes, where no significant difference in age at diagnosis was previously observed, we found that PLWH with lower healthcare encounter rates were diagnosed 2 years earlier than their counterparts.

\section{DISCUSSION}

The study's uniqueness centred around its populationbased nature and linkages between key administrative health databases which allowed for a comprehensive outlook on the healthcare utilisation of a large number of individuals over decades. Spanning over 12 years, this study underscored that, even in a setting where universal 
A. Alzheimer's and/or Non-HIV Dementia

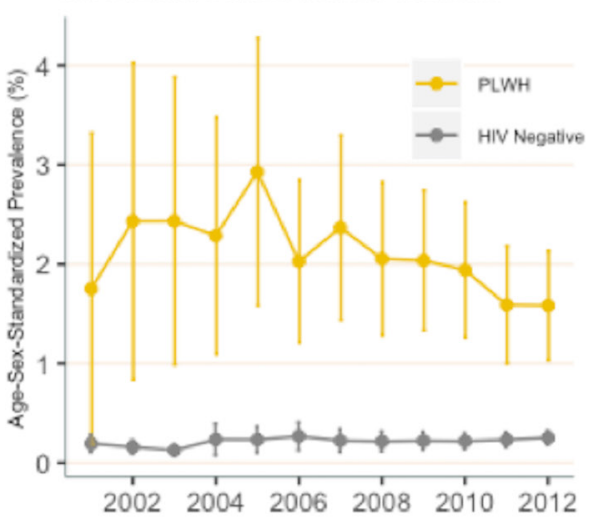

D. Cardiovascular Diseases

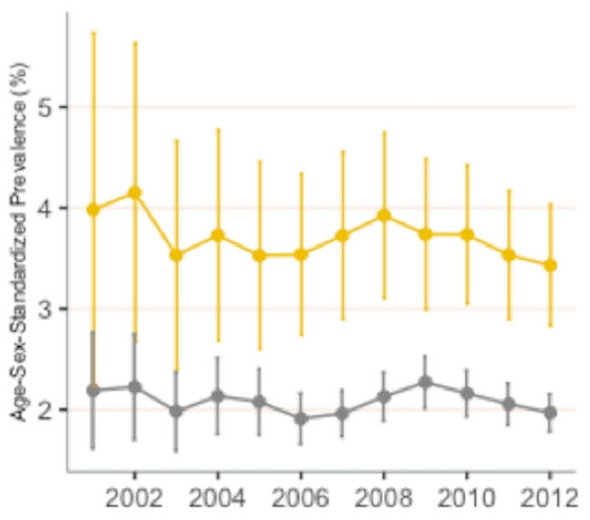

G. Kidney Diseases

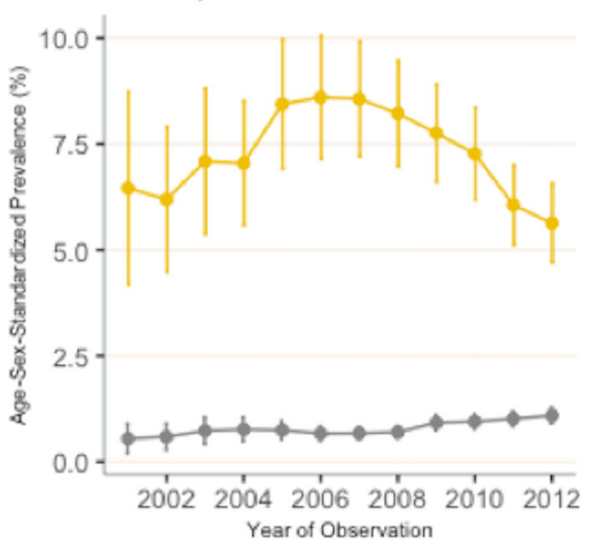

B. Non-AIDS-Defining Cancers

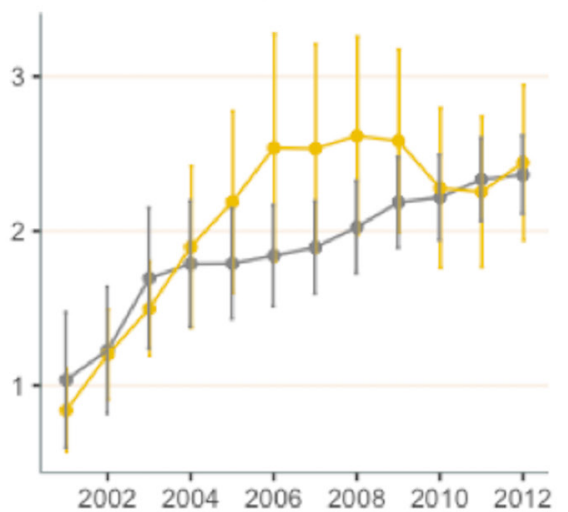

E. Diabetes Mellitus

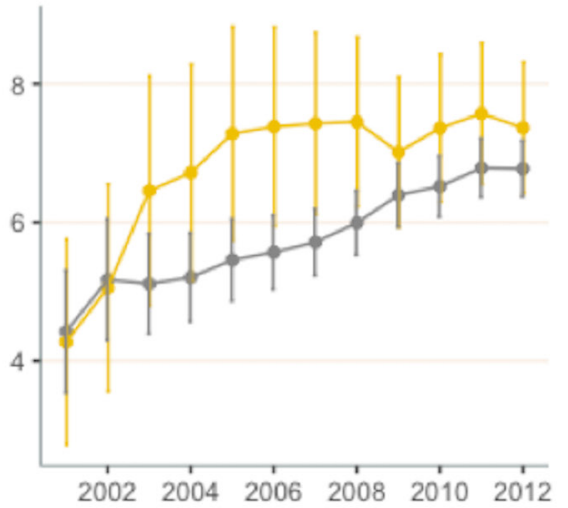

H. Liver Diseases

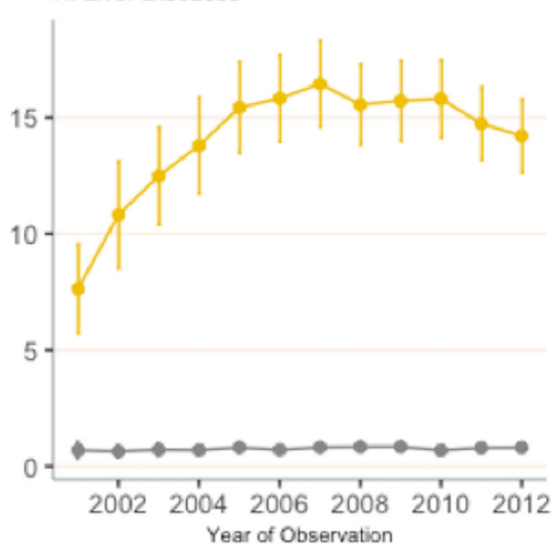

C. Chronic Obstructive Pulmonary Disease

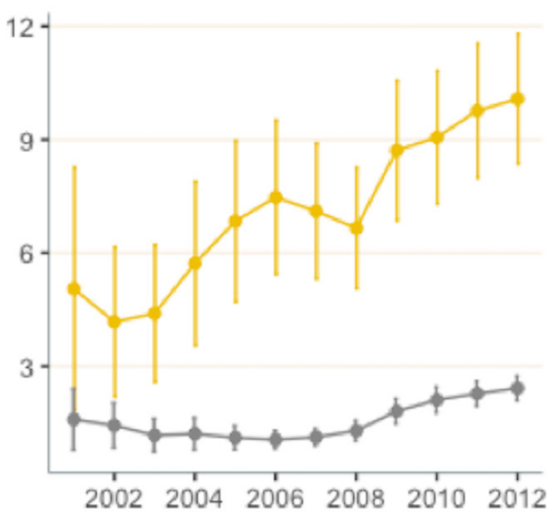

F. Hypertension
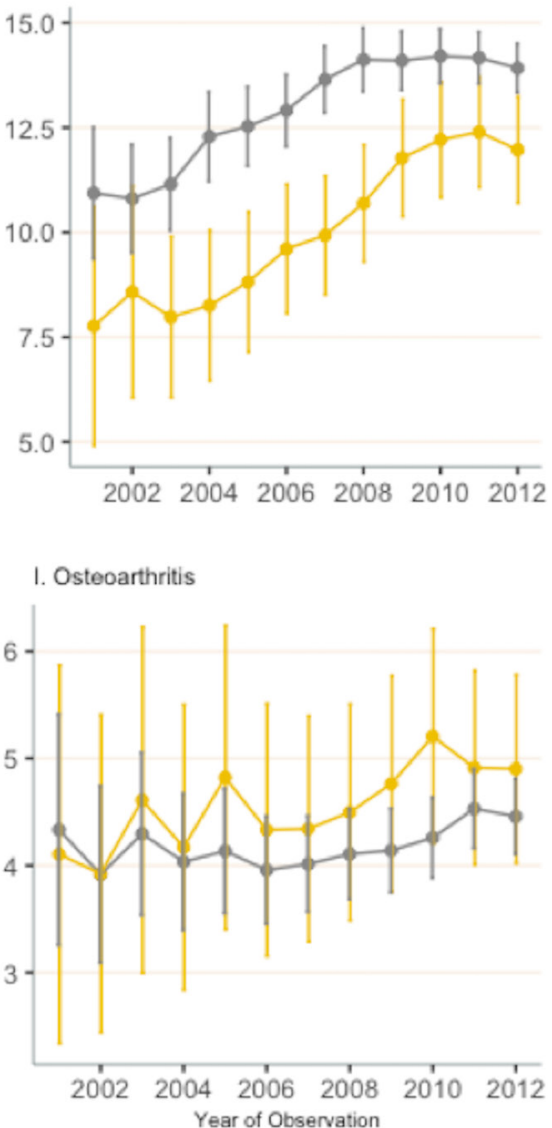

Figure 3 Annual trends of age-sex-standardised prevalence of selected comorbidities among PLWH and HIV-negative individuals across the study period (2001 to 2012) with 95\% confidence intervals. Note: PLWH, people living with HIV. Vertical scales differ for each graph for illustration purposes.

health coverage is available and after adjusting for differences in population distribution and healthcare utilisation, ART-treated PLWH are disproportionately affected by chronic age-associated diseases and, in some cases, at an earlier age than their age-sex-matched HIV-negative counterparts. While optimal ART has prolonged the lives of PLWH, their health outcomes remain compromised by the excess age-associated comorbidities. These findings highlight the need for integrated HIV care models that suit the evolving healthcare needs of ageing PLWH while reflecting the intersectionality of HIV and chronic diseases. Further, this study also contributes a thoughtful and replicable methodology that optimises identification of comorbidities using administrative data, and improves comparability of the observed disease burden within and between distinct study populations over time.

Our results are in concordance with past studies. ${ }^{91734-36}$ We found the prevalence of most chronic age-associated diseases (ie, Alzheimer's/dementia, COPD, CVD, kidney and liver diseases) were consistently higher among PLWH, especially those with an IDU history, compared with HIV-negative individuals. The statistically significant 
A. Alzheimer's and/or Non-HIV Dementia

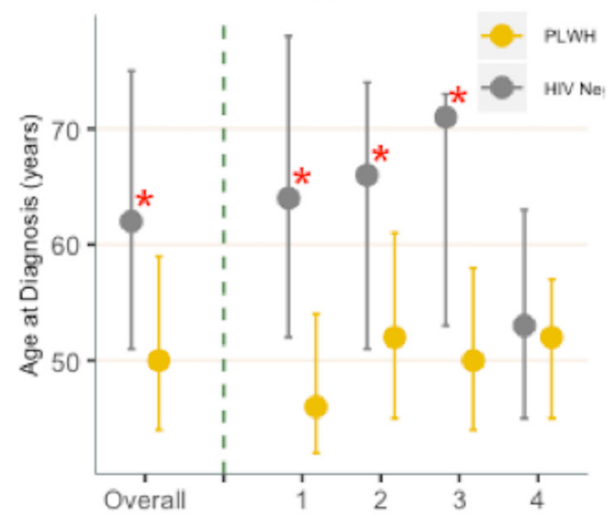

D. Cardiovascular Diseases

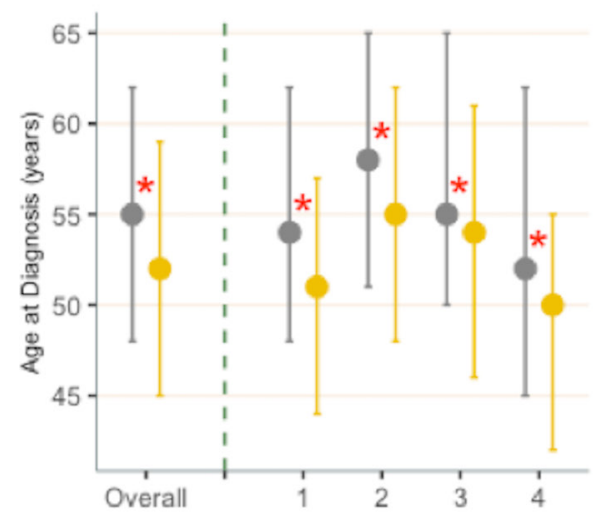

G. Kidney Diseases

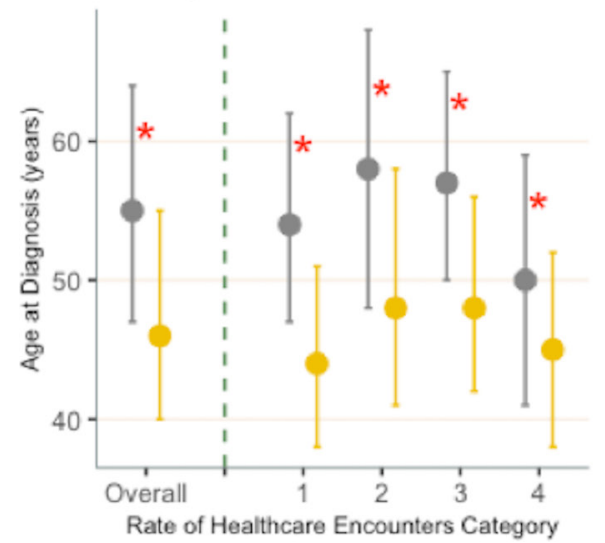

B. Non-AIDS-Defining Cancers

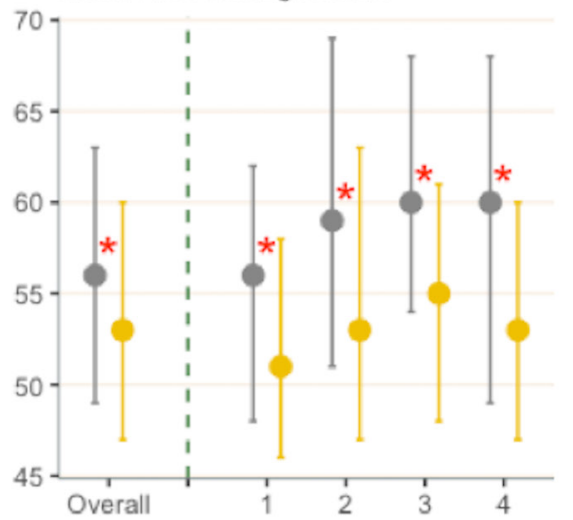

E. Diabetes Mellitus

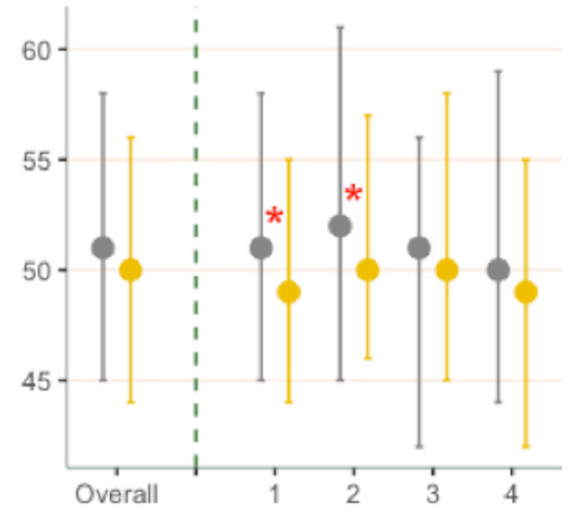

H. Liver Diseases

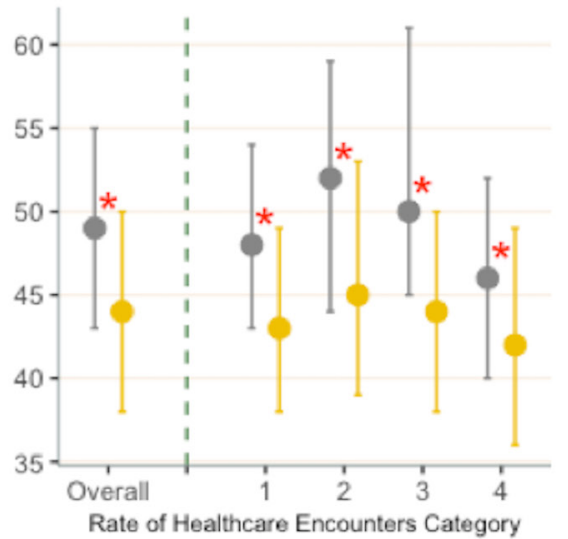

C. Chronic Obstructive Pulmonary Disease

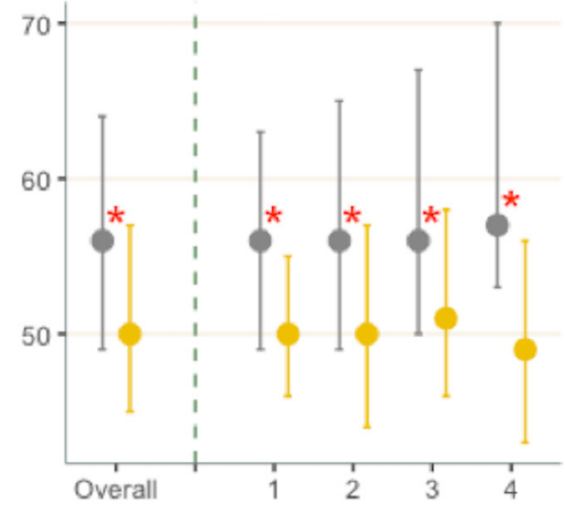

F. Hypertension

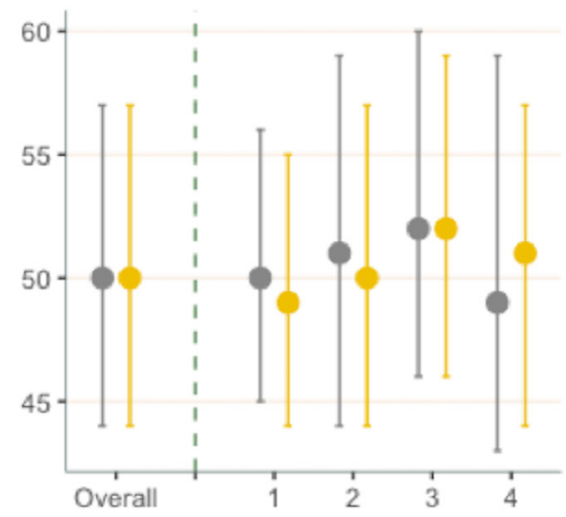

I. Osteoarthritis

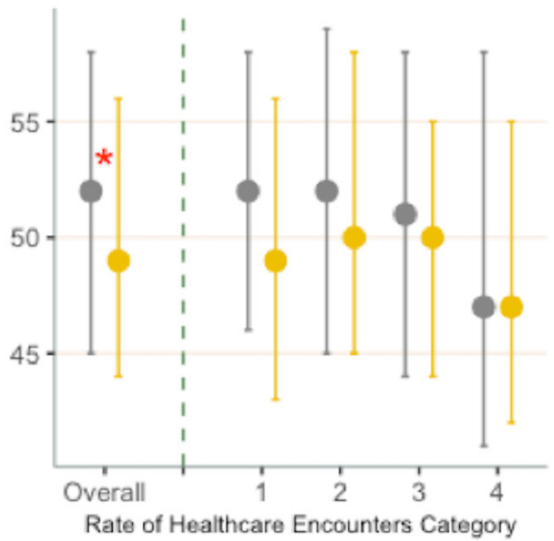

Figure 4 Distribution of median age at diagnosis of selected comorbidities with $25^{\text {th }}$ and $75^{\text {th }}$ percentiles; unadjusted (overall) and stratified by rate of healthcare encounters prior to the diagnoses. Note: PLWH, people living with HIV; HIV Neg, HIVnegative controls; rates of healthcare encounters prior to a diagnosis (measured in number of encounters/person-year) were categorised as lowest (1); lower (2); higher (3); and highest (4). ${ }^{*}$, significant difference in the median age at diagnosis for PLWH and HIV-negative controls ( $p$ value $<0.05$ ). Vertical scales differ for each graph for illustration purposes.

gaps observed between PLWH and HIV-negative controls may be explained by factors related to HIV infection and treatment, and by differences in the frequency of healthcare encounters and the presence of risk factors in both groups. For instance, tenofovir disoproxil fumarate and some ritonavir-boosted protease inhibitors, ${ }^{37}$ as well as hepatitis $\mathrm{C}$ co-infection, ${ }^{38}$ have been associated with increased risk of chronic kidney disease among PLWH. Alcohol use, which is consistently associated with HIV incidence ${ }^{39}$ has also been associated with an increased presence of cirrhosis biomarkers in HIV-hepatitis C co-infected adults. ${ }^{40}$ Similarly, higher prevalence of CVD among PLWH may be explained by the amplified presence of traditional risk factors (eg, smoking, excessive alcohol consumption and use of certain recreational drugs such as cocaine), which may be further exacerbated by HIV infection (eg, HIV-related inflammation) and treatment (eg, abacavir, tenofovir and dolutegravir 
have been associated with cardiovascular events, heart failure and weight gain, respectively). ${ }^{41} 42$ Among PLWH themselves, the observed disparities in the presence of several comorbidities between men and women support the growing literature, ${ }^{43-45}$ and demonstrate the importance of considering sex-specific preventative and treatment interventions in this population.

The prevalence of hypertension was higher among HIV-negative controls compared with PLWH throughout the study period. This observation agrees with reports from Norway and the USA suggesting that the prevalence of hypertension was not significantly different ${ }^{46}$ or higher among HIV-negative individuals compared with PLWH. ${ }^{18}$ Conversely, we observed apparent overlaps in the prevalence trends of non-AIDS-defining cancers, diabetes and osteoarthritis particularly in the early years of the study, followed by a steeper increase in the prevalence of these conditions among PLWH. Given the late onsets of both non-AIDS-defining cancers ${ }^{47}$ and osteoarthritis, ${ }^{48}$ these trends may likely be explained by the disproportionate presence of competing risks of death among PLWH in the earlier years of the study. Meanwhile, the trend in diabetes prevalence may be related to the use of early protease inhibitors and nucleoside reverse transcriptase inhibitors, which have been associated with an increased risk of diabetes among PLWH. ${ }^{49}$

Congruent with previous studies, ${ }^{9}{ }^{50}$ PLWH were diagnosed with each chronic age-associated comorbidity, except for diabetes and hypertension, at a significantly younger age than HIV-negative individuals. When compared exclusively to HIV-negative controls with similar healthcare encounter rates, PLWH were still diagnosed with these chronic comorbidities between 1 to 21 years earlier, suggesting that the observed differences in age at diagnosis between the two populations may be attributed to factors other than frequency of healthcare engagement. However, for some subgroups, including those diagnosed with Alzheimer's/dementia, diabetes and osteoarthritis, the observed early diagnoses among PLWH may be merely a consequence of PLWH's level of engagement with the healthcare system. Together, the observed earlier age at diagnosis and higher prevalence of several chronic age-associated comorbidities argue for an atypical ageing process among PLWH. ${ }^{13}{ }^{51}$ However, determining whether an accelerated or an accentuated ageing mechanism was involved likely depends on the specific comorbidities. For instance, the increasing prevalence trends of COPD and liver diseases among PLWH relative to HIV-negative individuals may imply that PLWH experienced an accelerated ageing mechanism, while the parallel prevalence trends of CVD and kidney diseases between the two groups may signal the involvement of an accentuated ageing mechanism. Nevertheless, further investigation, while controlling for key risk factors, is warranted to better understand the aetiology of the HIV-associated ageing process, and build a timely HIV care model tailored to this unique ageing population.
We should highlight that this study employed a more robust methodological approach compared with previous studies using the same cohort data and other studies using administrative health data. Based on preliminary analyses that explored the optimal lookback period to establish prevalent comorbidities in both PLWH and HIV-negative individuals using administrative data (results not shown), we elected to use a 5-year fix-sized lookback observation window to identify existing comorbidity diagnoses each year. This singular lookback observation window was selected to optimise detection of all 12 comorbidities, minimise sample restrictions due to insufficient follow-up and facilitate the comparison of annual prevalence within and between comorbidities. Correspondingly, to address in-migration and ensure the discrimination between pre-existing and new comorbidity diagnoses, individuals must have had at least 5 years of BC's administrative health records prior to baseline. In comparing the age at diagnosis of comorbidities between PLWH and HIV-negative individuals, we controlled for healthcare engagement level, a key confounder that prior studies have failed to consider. ${ }^{951}$ Lastly, whenever possible, we also used the published BC Ministry of Health's case definitions, which were largely consistent with the Canadian Chronic Disease Surveillance System case definitions and further informed by epidemiology and medical care experts considering BC-specific claims-related practices. As a consequence, readers must note that the identified comorbidities and prevalence estimates were specific to the current case-finding algorithms and observation measures, and comparisons with previous and future studies must be conducted with caution.

In addition to this study's methodological approach, our findings should also be interpreted in light of BC's highresource and universal healthcare context. For example, in 2009, the higher annual prevalence of hypertension among BC's PLWH (11.8\%), compared with PLWH from a large cohort in the USA $(8.7 \%),{ }^{19}$ may be partially explained by differences in health-seeking behaviours. By virtue of universal health coverage, BC's PLWH were likely more encouraged to access health services, which increased the likelihood of comorbidity diagnoses. With comparable healthcare systems, the difference in the prevalence of hypertension among PLWH in BC and male PLWH in Sweden in 2012 was minimal. ${ }^{52}$

Some limitations should also be considered. First, we used administrative health databases. While such data are an important source of information for empirical decision-making and medical research, they were not collected for research purposes and are prone to coding errors. However, as we used the same databases to ascertain the presence of comorbidities in both PLWH and HIV-negative controls, we assumed minimal differential misclassification bias across HIV status. Second, reliance on previously-collected administrative data rendered us unable to consider socioeconomic and lifestyle differences (eg, alcohol use and smoking) in our analyses. We attempted to address this through a subanalysis which 
revealed substantial differences in the prevalence of key comorbidities among PLWH with and without history of IDU. Third, the lack of ethnicity data among the HIVnegative controls and the high proportion $(\geq 40 \%)$ of unknown ethnicities among PLWH impeded our ability to fully assess ethnic disparities in the prevalence of these age-associated comorbidities. Lastly, the COAST study data are currently limited to March 2013. Once the updated COAST study data becomes available, the methodology established in this study can be readily applied to postulate the contemporary state of the disease burden in these populations. Nevertheless, the observed trends in prevalence and age at diagnosis provide valuable insights into the epidemiology of chronic age-associated comorbidities among PLWH, and support the prevention and management of these comorbidities as an increasingly important public health priority for PLWH and their healthcare providers.

In summary, our findings highlight that PLWH experience an excess burden of chronic age-associated comorbidities than their HIV-negative counterparts. The gaps in the prevalence of comorbidities between PLWH and their HIV-negative controls are likely multifactorial, as it may relate in part to HIV-related inflammation, socioeconomic and lifestyle differences and ART toxicities. Overall, these results stress the need for optimised general clinical screening for comorbidities at an earlier age among PLWH. Additionally, as the use of administrative data in health research has become increasingly common, our vigorous methodology to longitudinally measure and compare the prevalence and incidence of diseases in distinct populations is timely and readily reproducible to other settings. Future studies should build from this work, and focus on the development of evidence-based primary care guidelines that adapt to the changing healthcare needs of PLWH, integrate prevention and management of chronic diseases, and ultimately ensure healthy living and successful ageing in this population.

\section{Author affiliations}

${ }^{1}$ British Columbia Centre for Excellence in HIV/AIDS, Vancouver, British Columbia, Canada

${ }^{2}$ The University of British Columbia Faculty of Medicine, Vancouver, British Columbia, Canada

${ }^{3}$ VA Connecticut Health System, West Haven, Connecticut, USA

${ }^{4}$ Yale School of Medicine, New Haven, Connecticut, USA

${ }^{5}$ Arthritis Research Centre Of Canada, Richmond, British Columbia, Canada

${ }^{6}$ The University of British Columbia School of Population and Public Health, Vancouver, British Columbia, Canada

${ }^{7}$ Simon Fraser University Faculty of Health Sciences, Burnaby, British Columbia, Canada

Correction notice This article has been corrected since it first published. The provenance and peer review statement has been included.

Acknowledgements The authors would like to thank the BC-CfE, BC Ministry of Health, BC Vital Statistics Agency and the institutional data stewards for granting access to the data, and Population Data BC, for facilitating the data linkage process. We would like to thank Paul Sereda, Michelle Lu and Eric C Sayre for their contributions with methodologies of this study.

Contributors NGAN and VDL conceptualised and designed the study. JSGM, RSH and VDL curated the data and acquired funding. NGAN, HMT, JK and VDL constructed the methodology. NGAN and HMT performed data and other statistical analyses. NGAN and VDL wrote the original draft. NGAN, AP, HMT, MSJ, TM, ACJ, JK JSGM, RSH and VDL reviewed and edited the final draft. All authors have read and approved the final manuscript.

Funding This work was supported by the following sources of funding: COAST is funded by the Canadian Institutes of Health Research, through an Operating Grant (\#130419), a Foundation Award to RSH (\#143342) and support from the BC-CfE. JSGM's Treatment as Prevention (TasP) research, paid to his institution, has received support from Vancouver Coastal Health, the Public Health Agency of Canada, BC-Ministry of Health and US NIH (NIDA R01DA036307 and CTN 248); VDL is funded by a grant from the Canadian Institutes of Health Research (PJT-148595), by a Scholar Award from the Michael Smith Foundation for Health Research and a New Investigator award from the Canadian Institutes of Health Research. NGAN is supported by Canadian Institutes of Health Research Canada Graduate Student - Master's Award and the University of British Columbia Four-Year Doctoral Fellowship.

Disclaimer All inferences, opinions and conclusions drawn in this manuscript are those of the authors, and do not reflect the opinions or policies of the data stewards or the funders.

Competing interests JSGM's institutional grants have been provided by Gilead, J\&J, Merck and ViiV Healthcare.

Patient consent for publication Not required.

Ethics approval The University of British Columbia/Providence Health Care Research Ethics Board (H09-02905; H16-02036) and Simon Fraser University Office of Research Ethics (\#2013 s0566) provided ethics approval for this study.

Provenance and peer review Not commissioned; externally peer reviewed.

Data availability statement The British Columbia Centre for Excellence in HIV/ AIDS (BC-CfE) is prohibited from making this data set available publicly due to prohibitions in the information sharing agreement under which the data stewards provided the data to the $\mathrm{BC}$-CfE. The underlying analytical codes are available from the authors on request.

Supplemental material This content has been supplied by the author(s). It has not been vetted by BMJ Publishing Group Limited (BMJ) and may not have been peer-reviewed. Any opinions or recommendations discussed are solely those of the author(s) and are not endorsed by BMJ. BMJ disclaims all liability and responsibility arising from any reliance placed on the content. Where the content includes any translated material, BMJ does not warrant the accuracy and reliability of the translations (including but not limited to local regulations, clinical guidelines, terminology, drug names and drug dosages), and is not responsible for any error and/or omissions arising from translation and adaptation or otherwise.

Open access This is an open access article distributed in accordance with the Creative Commons Attribution Non Commercial (CC BY-NC 4.0) license, which permits others to distribute, remix, adapt, build upon this work non-commercially, and license their derivative works on different terms, provided the original work is properly cited, appropriate credit is given, any changes made indicated, and the use is non-commercial. See: http://creativecommons.org/licenses/by-nc/4.0/.

\section{ORCID iD}

Ni Gusti Ayu Nanditha http://orcid.org/0000-0002-3723-6418

\section{REFERENCES}

1 Deeks SG, Lewin SR, Havlir DV. The end of AIDS: HIV infection as a chronic disease. Lancet 2013;382:1525-33.

2 Samji H, Cescon A, Hogg RS, et al. Closing the gap: increases in life expectancy among treated HIV-positive individuals in the United States and Canada. PLoS One 2013;8:e81355.

3 May MT, Gompels M, Delpech V, et al. Impact on life expectancy of HIV-1 positive individuals of CD4+ cell count and viral load response to antiretroviral therapy. AIDS 2014;28:1193-202.

4 BC Centre for Excellence in HIV/AIDS. HIV Monitoring Quarterly Report for British Columbia - Fourth Quarter 2013, 2013. Available: http://www.cfenet.ubc.ca/sites/default/files/uploads/publications/ centredocs/bc-monitoring-report-13q4-updated-2015-jan-20.pdf [Accessed 15 Apr 2019].

5 BC Centre for Excellence in HIV/AIDS. HIV Monitoring Quarterly Report for British Columbia - Fourth Quarter 2018, 2018. Available: http://stophivaids.ca/qmr/2018-Q4/\#/bc

6 Wing EJ. Hiv and aging. Int J Infect Dis 2016;53:61-8. 
7 Antiretroviral Therapy Cohort Collaboration. Causes of death in HIV-1-infected patients treated with antiretroviral therapy, 19962006: collaborative analysis of 13 HIV cohort studies. Clin Infect Dis 2010;50:1387-96.

8 Lima VD, Lourenço L, Yip B, et al. Aids incidence and AIDS-related mortality in British Columbia, Canada, between 1981 and 2013: a retrospective study. Lancet HIV 2015;2:e92-7.

9 Guaraldi G, Orlando G, Zona S, et al. Premature age-related comorbidities among HIV-infected persons compared with the general population. Clin Infect Dis 2011;53:1120-6.

10 Eyawo O, Franco-Villalobos C, Hull MW, et al. Changes in mortality rates and causes of death in a population-based cohort of persons living with and without HIV from 1996 to 2012. BMC Infect Dis 2017;17:1-15.

11 Justice AC. Hiv and aging: time for a new paradigm. Curr HIVIAIDS Rep 2010;7:69-76.

12 Desquilbet L, Jacobson LP, Fried LP, et al. Hiv-1 infection is associated with an earlier occurrence of a phenotype related to frailty. J Gerontol A Biol Sci Med Sci 2007;62:1279-86 http://www. ncbi.nlm.nih.gov/pubmed/18000149

13 Sabin CA, Reiss P. Epidemiology of ageing with HIV. AIDS 2017;31:S121-8.

14 Pathai S, Bajillan H, Landay AL, et al. Is HIV a model of accelerated or accentuated aging? J Gerontol A Biol Sci Med Sci 2014;69:833-42.

15 Fisher M, Cooper V. Hiv and ageing. Curr Opin Infect Dis 2012;25:1-3.

16 Rassen JA, Bartels DB, Schneeweiss S, et al. Measuring prevalence and incidence of chronic conditions in claims and electronic health record databases. Clin Epidemiol 2019;11:1-15.

17 Rasmussen LD, May MT, Kronborg G, et al. Time trends for risk of severe age-related diseases in individuals with and without HIV infection in Denmark: a nationwide population-based cohort study. Lancet HIV 2015;2:e288-98.

18 Kong AM, Pozen A, Anastos K, et al. Non-HIV comorbid conditions and polypharmacy among people living with HIV age 65 or older compared with HIV-negative individuals age 65 or older in the United States: a retrospective claims-based analysis. AIDS Patient Care STDS 2019;33:93-103.

19 Wong C, Gange SJ, Moore RD. Multimorbidity among persons living with HIV in the U. S. Clin Infect Dis 2017:1-37.

20 Christensen S, Wolf E, Altevers J, et al. Comorbidities and costs in HIV patients: a retrospective claims database analysis in Germany. PLoS One 2019;14:e0224279.

21 Sutton SS, Magagnoli J, Cummings TH, et al. Chronic kidney disease, cardiovascular disease, and osteoporotic fractures in patients with and without HIV in the US veteran's Affairs administration system. Curr Med Res Opin 2019;35:117-25.

22 BC Centre for Excellence in HIV/AIDSop. Therapeutic guidelines antiretroviral (ARV) treatment of adult HIV infection, 2019. Available: http://cfenet.ubc.ca/sites/default/files/uploads/Guidelines/BC-CfEAdult-ARV-Therapeutic-Guidelines_2020-01-16.pdf [Accessed 10 Jan 2019].

23 British Columbia Centre for Excellence in HIV/AIDS (BCCfE). Drug treatment program, 2019. Available: http://www.cfenet.ubc.ca/ drug-treatment-program\%0Ahttp://www.cfenet.ubc.ca/research/ laboratory-program [Accessed 24 Apr 2018].

24 British Columbia Ministry of Health. Consolidation File (MSP Registration \& Premium Billing), 2014. Available: http://www.popdata bc.ca/data [Accessed 17 Feb 2019].

25 Canadian Institute for Health Information. Discharge Abstract database (Hospital separations), 2014. Available: http://www. popdata.bc.ca/data

26 British Columbia Ministry of Health. Medical services plan (MSP) payment information file, 2014. Available: http://www.popdata.bc.ca/ data [Accessed 17 Feb 2019].

27 BC Vital Statistics Agency. Vital statistics deaths, 2014. Available: http://www.popdata.bc.ca/data

28 BC Cancer Registry Data. Population data bc. PharmaNet, 2016.

29 BC Ministry of Health, 2014. Available: http://www.popdata.bc.ca/ data

30 Eyawo O, Hull MW, Salters K, et al. Cohort profile: the comparative outcomes and service utilization trends (coast) study among people living with and without HIV in British Columbia, Canada. BMJ Open 2018;8:e019115.

31 Chronic Disease Information Working Group. Bc chronic disease and selected procedure case definitions version 2017. last updated
2019;2015 http://www.bccdc.ca/health-professionals/data-reports/ chronic-disease-dashboard\#Case-Definitions

32 Statistics Canada. 2006 census of population. Ottawa, 2006. https:// www12.statcan.gc.ca/census-recensement/2006/dp-pd/tbt/Rp-eng $\mathrm{cfm}$ ? LANG $=E \& A P A T H=3 \& D E T A I L=0 \& D I M=0 \& F L=A \& F R E E=0 \& G C=0 \&$ $\mathrm{GID}=0 \& \mathrm{GK}=0 \& \mathrm{GRP}=1 \& \mathrm{PID}=88981 \& \mathrm{PRID}=0 \& \mathrm{PTYPE}=88971,97154 \&$ $\mathrm{S}=0 \& S H O W A L L=0 \& S U B=0 \&$ Temporal $=2006 \&$ THEME $=66 \& \mathrm{VID}=0 \&$ VNAMEE $=\&$ VNAMEF $=$

33 Lima VD, St-Jean M, Rozada I, et al. Progress towards the United nations 90-90-90 and 95-95-95 targets: the experience in British Columbia, Canada. J Int AIDS Soc 2017;20:e25011.

34 Mayer KH, Loo S, Crawford PM, et al. Excess clinical comorbidity among HIV-infected patients accessing primary care in US community health centers. Public Health Rep 2018;133:109-18.

35 Helleberg M, Pedersen MG, Pedersen CB. Associations between HIV and schizophrenia and their eff ECT on HIV treatment outcomes: a nationwide population-based cohort study in Denmark. Published Online First 2015.

36 Cook JA, Burke-Miller JK, Steigman PJ, et al. Prevalence, comorbidity, and correlates of psychiatric and substance use disorders and associations with HIV risk behaviors in a multisite cohort of women living with HIV. AIDS Behav 2018;22:3141-54.

37 Achhra AC, Nugent M, Mocroft A, et al. Chronic kidney disease and antiretroviral therapy in HIV-positive individuals: recent developments. Curr HIVIAIDS Rep 2016;13:149-57.

38 Rossi C, Raboud J, Walmsley S, et al. Hepatitis C co-infection is associated with an increased risk of incident chronic kidney disease in HIV-infected patients initiating combination antiretroviral therapy. BMC Infect Dis 2017;17:246.

39 Williams EC, Hahn JA, Saitz R, et al. Alcohol use and human immunodeficiency virus (HIV) infection: current knowledge, implications, and future directions. Alcohol Clin Exp Res 2016;40:2056-72.

40 Tsui Jl, Cheng DM, Libman $\mathrm{H}$, et al. Risky alcohol use and serum aminotransferase levels in HIV-infected adults with and without hepatitis C. J Stud Alcohol Drugs 2013;74:266-70 http://www.ncbi. nlm.nih.gov/pubmed/23384374

41 Venter WDF, Moorhouse M, Sokhela S, et al. Dolutegravir plus two different prodrugs of tenofovir to treat HIV. N Engl J Med 2019;381:803-15.

42 Choi Al, Vittinghoff E, Deeks SG, et al. Cardiovascular risks associated with abacavir and tenofovir exposure in HIV-infected persons. AIDS 2011;25:1289-98.

43 Palella FJ, Hart R, Armon C, et al. Non-Aids comorbidity burden differs by sex, race, and insurance type in aging adults in HIV care. AIDS 2019;33:2327-35.

44 Frazier EL, Sutton MY, Tie Y, et al. Differences by sex in cardiovascular comorbid conditions among older adults (aged 50-64 or $\geq 65$ years) receiving care for human immunodeficiency virus. Clin Infect Dis 2019;69:2091-100.

45 Willig AL, Westfall AO, Overton ET, et al. Obesity is associated with race/sex disparities in diabetes and hypertension prevalence, but not cardiovascular disease, among HIV-infected adults. AIDS Res Hum Retroviruses 2015;31:898-904.

46 Bergersen BM, Sandvik L, Dunlop O, et al. Prevalence of hypertension in HIV-positive patients on highly active retroviral therapy (HAART) compared with HAART-naïve and HIV-negative controls: results from a Norwegian study of 721 patients. Eur J Clin Microbiol Infect Dis 2003;22:731-6.

47 Smith DM, Salters KA, Eyawo O, et al. Mortality among people living with HIV/AIDS with non-small-cell lung cancer in the modern HAART era. AIDS Care 2018;30:936-42

48 Cope PJ, Ourradi K, Li Y, et al. Models of osteoarthritis: the good, the bad and the promising. Osteoarthritis Cartilage 2019;27:230-9.

49 Capeau J, Bouteloup V, Katlama C, et al. Ten-Year diabetes incidence in $1046 \mathrm{HIV}$-infected patients started on a combination antiretroviral treatment. AIDS 2012;26:303-14.

50 Shiels MS, Althoff KN, Pfeiffer RM, et al. Hiv infection, immunosuppression, and age at diagnosis of Non-AIDS-Defining cancers. CLINID 2016;269:ciw764.

51 De Francesco D, Wit FW, Bürkle A, et al. Do people living with HIV experience greater age advancement than their HIV-negative counterparts? AIDS 2019;33:259-68.

52 Jallow A, Ljunggren G, Wändell P, et al. Prevalence, incidence, mortality and co-morbidities amongst human immunodeficiency virus (HIV) patients in Stockholm County, Sweden - the Greater Stockholm HIV Cohort Study. AIDS Care 2015;27:142-9. 